https://doi.org/10.5719/aub-g/69.1/8

\title{
APPORTS DES SIG POUR LA GESTION DE LA SIGNALISATION ROUTIERE URBAINE ET LE COMPORTEMENT DES USAGERS DE LA ROUTE PAR RAPPORT AUX OBLIGATIONS ET INFORMATIONS
}

\author{
MOURAD ZITANI ${ }^{1}$, ABDELKADER ABDELLAOUI ${ }^{2}$
}

\begin{abstract}
Résumé
Il existe de nombreux travaux liés à une approche géographique de l'usage de la route, notamment pour la circulation routière, les transports urbains, le marquage routier, les sentiers récréatifs, le réseau cyclable, la gestion des chaussées ou encore les accidents de la route. Les travaux sur la signalisation routière urbaine sont au contraire très rares.

Cette signalisation demeure pourtant à la fois le principal outil de gestion de la voirie mais aussi l'unique moyen d'orienter l'usager de la route sur la manière de faire quand il est au volant, voire à pieds. Le suivi permanent de l'état de cette signalisation est important pour éviter la congestion du trafic ainsi que les accidents de la circulation. Une approche géographique de ce suivi, par le moyen des systèmes d'informations géographiques, devrait permettre une vision globale et un contrôle quasiment en temps réel.

En Algérie la gestion de la signalisation routière est encore réalisée par les moyens traditionnels où l'outil numérique n'est qu'accessoire. On trouve peu de travaux de recherche sur cette question.

L'objectif du présent travail est double : i) réaliser un état des lieus de la signalisation routière dans la ville pour tenter de comprendre le comportement social vis-à-vis de cette signalisation ii) proposer un avant-projet de réseau de signalisation urbaine de la ville de Laghouat (Algérie), s'appuyant sur l'utilisation des systèmes d'informations Géographiques "SIG » comme outil d'aide à la gestion et la mise en œuvre d'une stratégie de suivi du patrimoine de signalisation ; nous nous limitons dans le cadre de ce travail à la signalisation verticale. Dans le modèle proposé, nous avons introduit le comportement des usagers par rapport aux signalisations, notamment le respect des indications. Une enquête particulière a été menée sur terrain.
\end{abstract}

Mots clés : signalisation routière, Laghouat, organisation urbaine, usager de la route.

1 Université de Laghouat, Algérie.

2 Réseau Campus Virtuel Avicenne, Paris, abdellaoui.geo@gmail.com 
There are many works related to a geographic approach to road use: road traffic, urban transport, road markings, recreational trails, the bicycle network, roadway management or road accidents. On the contrary, there is little work on urban road signs.

However, this signage remains both the main road management tool but also the only way to guide the road user on how to do it while driving or even on foot. Continuous monitoring of the status of this signage is important to avoid traffic congestion and traffic accidents. A geographic approach to this monitoring, by means of geographic information systems, should allow a global vision and control almost in real time.

In Algeria, the management of road signs is still carried out by traditional means where the digital tool is only an accessory. There is little research on this issue.

The objective of this work is twofold: i) carry out an inventory of road signs in the city to try to understand social behavior towards this signaling; ii) propose a draft urban signaling network for the city of Laghouat (Algeria) based on GIS system to manage road signs and elaborate a signaling heritage monitoring strategy; we limit ourselves in the context of this work to vertical signage.

In the proposed model, we have introduced the behavior of users with regard to signals, in particular respect for indications. A specific investigation was carried out in the field. A specific investigation was carried out in the field.

Keywords: road signs; Laghouat; urban organization; road users.

\section{Introduction}

La signalisation demeure à la fois le principal outil de gestion de la voirie mais aussi l'unique moyen d'orienter l'usager de la route sur la manière de faire quand il est au volant, voire à pieds. Le suivi permanent de l'état de cette signalisation est important pour éviter la congestion du trafic ainsi que les accidents de la circulation. Une approche géographique de ce suivi, par le moyen des systèmes d'informations géographiques, devrait permettre une vision globale et un contrôle quasiment en temps réel.

Il existe de nombreux travaux liés à une approche géographique de l'usage de la route, notamment pour la circulation routière, les transports urbains, le marquage routier, les sentiers récréatifs, le réseau cyclable, la gestion des chaussées ou encore les accidents de la route. Les travaux sur la signalisation routière urbaine sont au contraire très rares. 
APPORTS DES SIG POUR LA GESTION DE LA SIGNALISATION ROUTIÈRE URBAINE ET LE COMPORTEMENT DES USAGERS DE LA ROUTE PAR RAPPORT AUX OBLIGATIONS ET INFORMATIONS

Parmi ces travaux, nous pouvons signaler ceux qui nous paraissent les plus proches de notre travail. Emery, J. et al. (2014) s'interrogent "sur l'opportunité qu'offre la simulation multi-agents pour intégrer des données d'observation du trafic routier au sein d'un SIG ». L'application est faite sur la ville de Dijon (France). Pellecuer et al. (2009) notent que «la sécurité des usagers de la route figure parmi les priorités du ministère des Transports du Québec (MTQ). Dans ce cadre, le maintien de la visibilité du marquage routier en tout temps et sous toutes les conditions climatiques est bien évidemment primordial. L'amélioration de la signalisation horizontale et de sa gestion s'avère donc être une composante essentielle du service offert par l'administration routière aux usagers de la route». Le travail Moretti S-D (2018) porte sur la gestion et le partage des données des sentiers récréatifs canadiens et une nouvelle norme des données intégrées à un SIG. Il a pour objectif le "développement d'un schéma d'application GML (Geographic Markup Language) dédié à la gestion et à la diffusion des données géographiques et non géographiques relatives aux sentiers récréatifs ».

Dupuis, B. (2005) s'est proposée de décrire les processus de gestion des supports d'affichage culturel, officiel et commercial, sur le domaine public de la Ville de Neuchâtel. Mentfakh, T. et al. (2014), partant du constat d'augmentation brusque des véhicules en Algérie qui « engendre des désorganisations dans la gestion courante comme la modification permanente de la structure des trafics et des usages sur les réseaux routiers", affirment "qu'il faudrait établir régulièrement un plan de signalisation routière structurée » et proposent la réalisation d'un Système d'Information Géographique pour la signalisation du réseau routier dans le cas pratique la région de Mostaganem.

Le Conseil Général 'Loire' a mis sur site un rapport (non daté) sur la «Mise en place d'un SIG pour la gestion des équipements sur les routes départementales de la Loire ». Le projet visé a pour objet «Élaboration du schéma directeur de signalisation directionnelle et touristique ».

Rappelons que la signalisation urbaine est l'outil de communication par excellence entre la route et ses usagers; elle se compose de trois éléments qui interagissent entre eux, à savoir: i) la route et son environnement, ii) la circulation et les véhicules qui en font partie, et enfin iii) les usagers en voiture, éventuellement à vélo et les piétons. Elle 
est principalement composée de panneaux, de marques sur la chaussée et de signaux lumineux et permet à l'usager d'adapter sa conduite aux particularités présentes sur la route. La signalisation agit ainsi comme le mode d'emploi de la route, en édictant des prescriptions, en signalant des dangers et en donnant des renseignements sur comment et où se diriger pour se rendre à destination. Elaborée et suivi par des services particulièrement dédiés, elle constitue un lien important avec l'usager de la route et doit, à cet effet, faire l'objet d'un regard attentif permanent.

En Algérie la gestion de la signalisation routière est encore réalisée par les moyens traditionnels où l'outil numérique n'est qu'accessoire. On trouve peu de travaux de recherche sur cette question.

Le présent travail a pour objectif du présent travail est double : i) un état des lieus de la signalisation routière dans la ville pour tenter de comprendre le comportement social vis-à-vis de cette signalisation ii) proposer un avant-projet de réseau de signalisation urbaine de la ville de Laghouat (Algérie), s'appuyant sur l'utilisation des systèmes d'informations Géographiques «SIG » comme outil d'aide à la gestion et la mise en œuvre d'une stratégie de suive du patrimoine de signalisation ; nous nous limitons dans le cadre de ce travail à la signalisation verticale.

\section{Présentation de la zone d'étude}

La ville de Laghouat (Algérie), chef-lieu de wilaya (département), est située à $400 \mathrm{~km}$ au sud d'Alger sur le grand axe routier Nord-Sud à une altitude de $750 \mathrm{~m}$ sur le flanc sud de l'Atlas saharien. Elle se présente sous la forme de deux amphithéâtres qui se font face, sur les flancs de deux mamelons du djebel Tizigarine allongés dans le sens Nord-Est au Sud-Ouest.

D'oasis construite sur les deux flancs du djebel Tizigarine, Laghouat s'est transformée en véritable métropole où le construit a $\mathrm{d}$ 'abord pris la place des jardins puis s'est étendu dans une première phase en suivant la RN1.

A cet effet, Abdellaoui (2007) note que «les vieux quartiers constituaient le centre de vie de la ville (résidences, commerces, rencontres), ils sont désormais petit à petit désertés et délaissés ; même 
APPORTS DES SIG POUR LA GESTION DE LA SIGNALISATION ROUTIÈRE URBAINE ET LE COMPORTEMENT

DES USAGERS DE LA ROUTE PAR RAPPORT AUX OBLIGATIONS ET INFORMATIONS

dans les cas où certains petits commerces tentent de braver les mutations et ou un noyau de vie semble se maintenir, on sent une sorte d'abandon des propriétés pour des multiples raisons ».

La population de Laghouat est passée de 42800 hab en 1977 à 119043 hab en 2003, soit une multiplication par un facteur de 2.8 en 26 ans (Benblidia et al, 2006); en 2011 elle était de 144747 hab selon les statistiques de l'Office National des Statistiques (ONS) ; elle sera en 2030 de 196122 hab selon des estimations.

Benkouider et al. (2012) notent que la ville de Laghouat occupe une position stratégique en tant que ville relai, entre le Nord et le Sud et représentant le point de convergence des anciens parcours de caravanes. Elle est située à $400 \mathrm{~km}$ au sud d'Alger et à une altitude moyenne de $751 \mathrm{~m}$. Elle se développe de l'Est à l'Ouest sur trois collines, sorte d'arête rocheuse entre une plaine au nord et une daya au sud.

Rappelons enfin que l'exploitation et l'entretien du réseau routier de la ville sont confiés au service d'exploitation de la direction des travaux publics (DTP) de la wilaya dont la mission consiste à offrir aux usagers de la route un niveau de service satisfaisant de confort et de garantir la sécurité routière grâce à l'entretien et la préservation du patrimoine routier ainsi que les dépendances de la route (équipements de signalisation, éclairage...).

\section{Méthodes et outils}

\subsection{Hypothèses et démarches}

Le présent travail concerne de manière particulière la vile de Laghouat mais la démarche peut être étendue à toute autre agglomération urbaine. Nous nous limitons ici à la signalisation verticale à l'intérieur de la ville et à sa proximité; ce type de signalisation regroupe l'ensemble des signaux conventionnels implantés verticalement dans le champ visuel de l'usager de la route; dans le cadre de ce travail nous nous sommes limités aux types de panneaux présents dans la région d'étude. Nous avons ainsi seize types de signalisation verticale résumés par le tableau (1). L'application à 
d'autres zones urbaines comportant plus ou moins de types de panneaux de signalisation peut être réalisée en ajoutant des couches d'informations supplémentaires. Une couche panneau publicitaire est également inclue; elle ne fait pas communément partie de la signalisation routière gérée par les services administratifs compétents mais fait partie du cadre visuel de l'utilisateur de la route.

Une couche « routes » a également été créée.

Nous avons également réalisé des enquêtes terrain pour apprécier le comportement des usagers de la route face à la signalisation routière ; pour cela, nous avons ciblé des points d'implantation d'un certain nombre de signaux et avons décompté les respects et non respects des indications.

\subsection{Données et outils}

\subsubsection{L'image satellitale}

Nous avons utilisé l'application (maps downloader for Google satellite) dans sa version gratuite, pour acquérir une image Google Earth de très bonne résolution de la ville de Laghouat que nous avons calé sous MapInfo ; une image de Google Earth est également utilisée après calage avec la projection 'Universal Transverse Mercator WGS84 zone 31N'.

\subsubsection{Les données terrain}

2.2.2.1. Les panneaux de signalisation verticale : Nous avons trouvé 16 types de signalisation verticale à Laghouat comme le montre le tableau (1).

Tableau 1

Les panneaux de signalisation verticale à Laghouat

\begin{tabular}{|c|c|c|}
\hline Intitulé du panneau & Type & Nombre de panneaux \\
\hline Passage pour piétons & A 2b & 22 \\
\hline Ralentisseurs & A 7 & 33 \\
\hline Annonce d'un giratoire & AB 25 & 13 \\
\hline Signal d'intersection & A 22 & 22 \\
\hline
\end{tabular}


APPORTS DES SIG POUR LA GESTION DE LA SIGNALISATION ROUTIÈRE URBAINE ET LE COMPORTEMENT DES USAGERS DE LA ROUTE PAR RAPPORT AUX OBLIGATIONS ET INFORMATIONS

\begin{tabular}{|c|c|c|}
\hline Marquer un arrêt (STOP) & B 2 & 34 \\
\hline Cédez le passage & B 1 & 37 \\
\hline Interdiction de tourner & C7 et C8 & 7 \\
\hline Interdiction d'accès à tout véhicule & C1 & 23 \\
\hline Limite de vitesse & C11a & 58 \\
\hline Interdiction de stationnement & C11a & 13 \\
\hline Indication du sens de rotation obligatoire & D & 16 \\
\hline Identification de route & E & 75 \\
\hline Arrêt d'autobus & & 32 \\
\hline Feux tricolores & & 4 \\
\hline Indication de noms de quartiers & & 21 \\
\hline Panneaux publicitaires & & 19 \\
\hline Nombre total de panneaux & & 429 \\
\hline
\end{tabular}

Nous avons relevé par GPS les coordonnées géographiques des 429 panneaux ; les informations ont été portées sur fichier Excel. Nous avons ensuite créé sous MapInfo 16 tables correspondant à ces types de signalisations verticales puis créé les points correspondants en utilisant les coordonnées géographiques relevées.

A ces 16 tables nous avons ajouté deux tables:

- Une table 'route' sur laquelle sont dessinées en polylignes les trois catégories de routes : i) la route nationale (RN1), ii) les départementales et les routes à l'intérieur de l'agglomération.

- Une table 'secteurs urbains' qui constituent les « grands quartiers »

Le fond de carte est constitué par un extrait d'image satellitale Google Earth que nous avons géoréférencée.

2.2.2.2. Enquêtes sur le comportement des usagers de la route: Nous avons mené une enquête terrain en date du 13 septembre 2018 pour relever le comportement des usagers sur des points d'intérêt que nous avons choisis pour les signaux particuliers suivants : i) STOP, ii) passage pour piétions, iii) cédez le passage. Pour chacun de ces trois types de signaux, nous avons relevé le pourcentage de respect du signal par l'usager pour 33 points d'observation pour le signal 'stop', 32 points pour le signal 'passage pour piétons' et 37 points pour le signal 'céder le passage'. Nous avons noté les coordonnées de l'ensemble des points d'observation par GPS ; ceci nous a permis de les localiser et constituer des couches correspondantes sous MapInfo. 

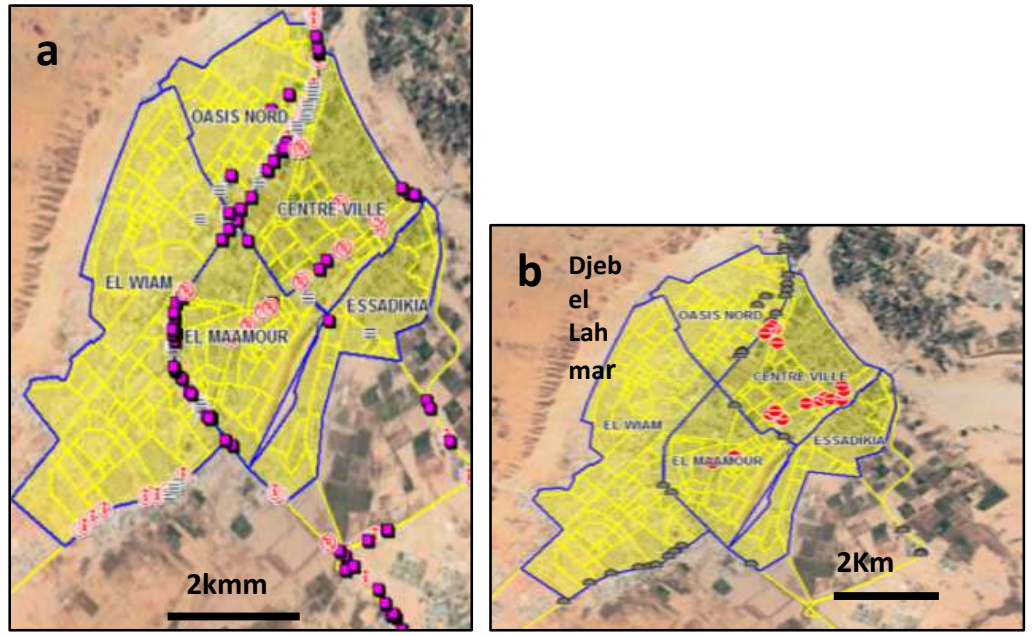

Fig. 1. Exemples de résultats (Sources : Auteurs)

\subsubsection{Les outils logiciels}

Pour l'élaboration de notre base de données géoréférencées, nous avons fait le choix de l'outil SIG MapInfo; nous avons travaillé avec la version 8.5 dont nous disposons d'une licence. Le choix de MapInfo a été largement influencé par la facilité d'apprentissage des agents de l'administration pour cet outil.

L'outil Excel (office pro 2010 avec licence) est utilisé pour la collecte des données terrain et leur migration vers MapInfo.

\section{Principaux résultats}

La figure (1) montre deux exemples de résultats: en (a) nous montrons les panneaux de direction obligatoire, de limite de vitesse et de passage pour piétons; en (b) seuls les passages pour piétons sont visualisés. Cet exemple montre les possibilités d'usage de la base de données géoréférencées : concentration sur un panneau de signalisation particulier ou analyse d'une combinaison de panneaux de signalisation au gré de l'utilisateur, en fonction d'un problème à traiter. 

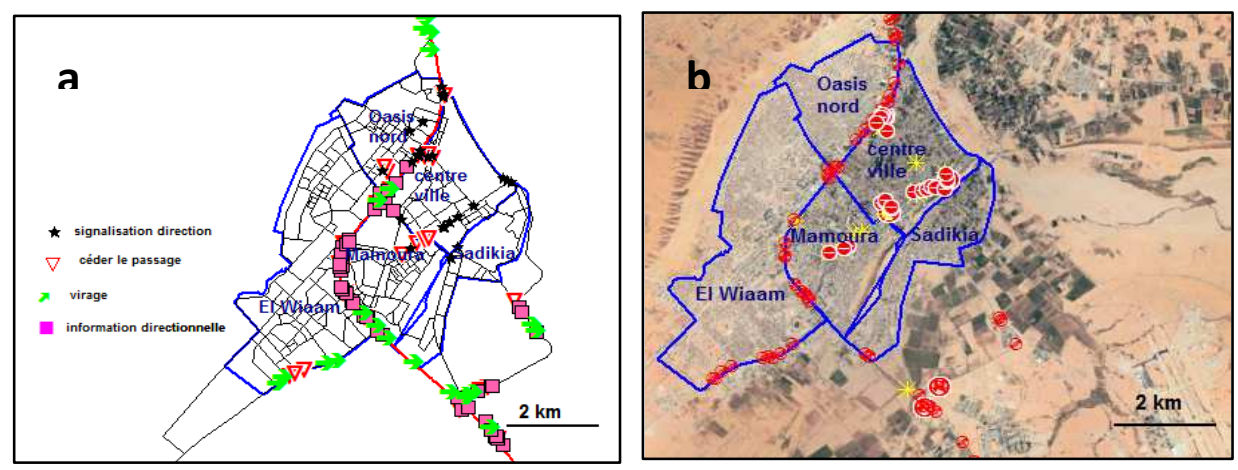

Fig. 2. Les panneaux par grands types ; (a) les obligations ; (b) les interdictions (Sources : figures issues des traitements sur MapInfo réalisés par les auteurs)

La figure (2) montre les panneaux signalant une obligation (a) et les interdictions (b) ; en (a) sont représentés les signalisations concernant : le céder le passage, l'obligation de tourner, ainsi que des informations directionnelles; en (b) nous avons visualisé les signalisations de stationnement interdit (symbole en couleur jaune pour le différencier), de sens interdit, $\mathrm{d}$ 'interdiction de tourner et limite de vitesse.

La figure (3) nous montre :

- en (a) les panneaux signalétiques concernant: les passages piétons, les ralentisseurs, les feux tricolores et les ronds-points

- en (b) : les panneaux publicitaires et les arrêts d'autobus

Une requête SQL nous a permis de déterminer les superficies ainsi que le nombre total de panneaux de signalisation routière des cinq zones principales de la ville que nous présentons sur le tableau (1).

Superficies des quartiers

\begin{tabular}{|c|c|c|}
\hline Quartier & Superficie en $\mathbf{k m}^{\mathbf{2}}$ & Nombre de signaux \\
\hline Centre-ville & 4.05 & 89 \\
\hline El Mamourah & 3.54 & 101 \\
\hline Essadikia & 2.88 & 15 \\
\hline El Wiaam & 8.58 & 84 \\
\hline Oasis Nord & 3.08 & 68 \\
\hline
\end{tabular}



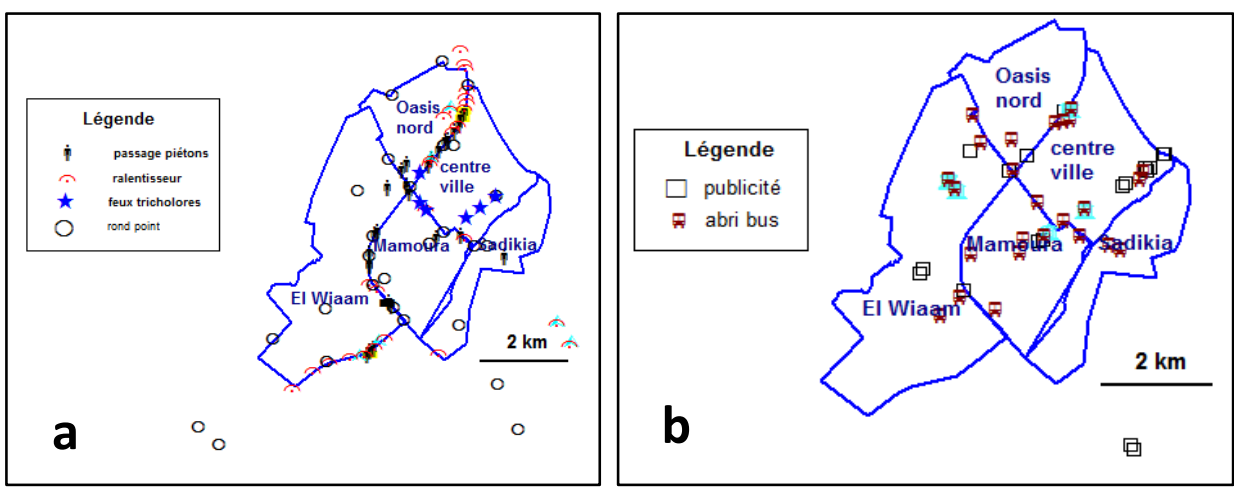

Fig. 3. Autres signalisations particulières

Le centre-ville est la principale zone d'activité commerciale. Essadikia est la zone qui avait accueilli la sédentarisation des nomades pendant l'époque coloniale. Oasis Nord représente la première extension nord de la ville. El Wiaam représente une sorte de nouvelle ville.

Le résultat des enquêtes sur le respect des signalisations routières verticales est donné par le tableau (2) :

Tableau 2

\section{Respect des signalisations routières}

\begin{tabular}{|c|c|c|c|c|c|c|c|}
\hline \multicolumn{3}{|c|}{$\begin{array}{c}\text { stop : } \\
33 \text { signaux } \\
\end{array}$} & \multicolumn{3}{|c|}{$\begin{array}{c}\text { passage piétions : } \\
32 \text { signaux } \\
\end{array}$} & \multicolumn{2}{|c|}{$\begin{array}{c}\text { céder le passage : } \\
37 \text { signaux }\end{array}$} \\
\hline 100 & $70-90$ & $<70$ & 100 & $50-90$ & $<50$ & 100 & $60-90$ \\
\hline 8 & 15 & 10 & 0 & 21 & 11 & 13 & 24 \\
\hline $24.2 \%$ & $45.5 \%$ & $30.3 \%$ & $0.0 \%$ & $65.6 \%$ & $34.4 \%$ & $35.1 \%$ & $64.9 \%$ \\
\hline
\end{tabular}

L'enquête a été réalisée le sur 33 panneaux pour le signal stop, 32 panneaux pour le signal passage piétons et 37 panneaux du signal céder le passage. Le tableau (2) nous fournit le pourcentage de respect du panneau par le conducteur (ligne 2) et le nombre de point de contrôle (ligne 3) pour chaque catégorie de pourcentage de respect ; il représente une compilation des résultats dans laquelle nous avons retenu trois classes pour les deux panneaux 'Stop' et 'passage piétons' et seulement deux classes pour le panneau 'céder le passage'. 
APPORTS DES SIG POUR LA GESTION DE LA SIGNALISATION ROUTIÈRE URBAINE ET LE COMPORTEMENT

DES USAGERS DE LA ROUTE PAR RAPPORT AUX OBLIGATIONS ET INFORMATIONS

\section{Discussions}

La figure (1) nous montre que l'outil permet de visualiser les résultats selon le type de problème à traiter: type particulier de panneaux à surveiller dans l'ensemble de la cité ou analyse spécifique à un quartier. Ceci est d'une utilité pour les services administratifs ainsi que pour d'éventuels travaux de recherches sur la cité. Il est évident que le modèle proposé peut être adapté à d'autres zones d'études, sous réserves du travail de collectes d'informations particulières.

Des figures (2) et (3), nous noterons les points essentiels suivants :

- La concentration de panneaux de signalisation sur le route nationale 1 (RN1); cela pourrait sembler normal à cause du double usage de cette voie : i) son caractère de voie de passage entre le Nord et le Sud et ii) l'intégration de cette voie dans l'activité de la cité (proximité des commerces, annexes administratives et habitations et donc de traversée par obligatoire par les piétons.

- La concentration de panneaux de signalisation dans le centreville en opposition aux quartiers 'Wiaam' qui est quasiment l'équivalent d'une nouvelle ville encore en cours de réalisation et du quartier 'Essadikia', traditionnellement considéré comme quartier « secondaire » peu aménagé par rapport au reste de la ville.

- Les panneaux 'abri bus' sont certes concentrés sur le centre-ville mais sont également présents sur les parties reculées des quartiers périphériques ; cela montre que le réseau de transport urbain suit assez bien le développement de la ville.

- Le nombre de ronds-points est relativement important, en particulier sur la RN1, mais souvent au-delà.

- Le nombre de ralentisseurs de la circulation (dos-d'âne) est relativement important; nous avons constaté que ces ralentisseurs n'obéissent pas aux normes conventionnelles (hauteur, accès et sortie, signalisation anticipée)

- La figure ( $3 b)$ nous indique la présence de nombreux panneaux de publicité dans les grandes zones (excepté 'Essadikia'); ceci est nouveau dans la culture populaire.

Le tableau (1) nous donne à la fois les superficies des grands quartiers mais aussi le nombre de signaux dans chaque zone. Il aurait été intéressant de rapporter ce nombre à la population et à l'activité 
commerciale (voire artisanale) de chaque quartier ; nous n'avons pas pu trouver de telles statistiques. Nous noterons cependant que le plus grand nombre de panneaux de signalisation se trouve dans le quartier 'Mamourah' qui devait être, à l'origine, un quartier résidentiel mais qui a reçu l'implantation de diverses activités administratives, commerciales et artisanales (grande annexe de la poste, tribunal, banques, commerces de proximités, restaurants, café, bazars, officines et cabinets de santé publique). Ceci explique la densité des fréquentations des usagers et donc du nombre important de panneaux règlement la circulation routière (101). Ce tableau nous indique aussi que le nombre de panneaux de signalisation routière n'est que de 15 dans le quartier Essadikia, soit $4.2 \%$ du nombre total de panneaux ; ceci confirme encore une fois le caractère de traitement « particulier » de ce quartier.

Le tableau (2), résultat de notre enquête terrain sur le respect de la signalisation routière par les conducteurs, nous informe que :

- Le pourcentage de respect de la signalisation est globalement faible (moins de $50 \%$ pour l'ensemble des panneaux contrôlés)

- Le respect strict n'est que de $24 \%$ pour le signal 'stop' et $35 \%$ pour 'céder le passage' ; il est de $0 \%$ pour le 'passage piéton' ; la culture de la priorité au piéton sur l'usage de la route n'est pas encore introduite dans la conscience populaire

- Le signal 'céder la passage' est peu respecté

- Même le signal 'stop' qui nécessite de marquer un arrêt obligatoire est très peu respecté dans cette forme

- Ces remarques nous montrent qu'une campagne de sensibilisation de l'usager de la route suivie de consignes aux écoles de conduite doit être mise en place.

\section{Conclusion}

Le présent travail nous a permis de proposer un modèle de gestion la signalisation routière verticale utilisant des enquêtes terrain sur le comportement des conducteurs de véhicules et une base de données géoréférencées sur l'ensemble des signalisations routières verticales à Laghouat. Nous avons utilisé l'outil logiciel "MapInfo" pour deux 
raisons principales : la disposition d'une licence et le fait que certains services publics locaux l'utilisent ce qui facilite le dialogue. L'usage de MapInfo dans ce travail n'est évidemment pas exclusif. Une migration vers l'outil free 'Qgis' est possible et ne pose pas de problème particulier.

Ce modèle est bien évidemment d'une grande utilité pour les gestionnaires du réseau de signalisation (services de la DTP, en particulier); mais il peut être mis à la disposition des écoles de conduite en leur fournissant un outil supplémentaire d'appui à la formation en montrant l'ensemble des signalisations verticales dans la ville et en sensibilisant les apprenants sur le comportement des usagers de la route.

Les résultats que nous avons présentés nous ont permis d'avoir une idée de répartition des panneaux de la circulation routière par quartier et d'en déduire, par voie de conséquence, la dynamique de chaque quartier ou zonage de la ville.

Nous avons également pu apprécier les comportements des conducteurs par rapports aux signalisations ; nous avons ainsi pu constater le pourcentage élevé du non-respect des obligations ; ce qui nécessiterait des campagnes de formation et de sensibilisation particulières.

A ce travail manque l'absence de statistiques de population globales et par zones d'habitation et d'activités; un travail d'enquêtes supplémentaire pour pallier à cette carence sort du cadre de cette étude mais aurait permis d'aller plus loin dans les analyses.

\section{BIBLIOGRAPHIE}

Abdellaoui, Abdelkader (2007), Intégration de l'imagerie satellitale multi-résolution et de données terrain pour la réhabilitation des quartiers anciens en milieu oasien. Cas de la ville de Laghouat (Algérie); Analele Universitatii București ; Geografie ; 2007.

Benblidia, Nadjia, Abdelkader Abdellaoui et Abdelkrim Bensaid (2006), Utilisation de la morphologie mathématique pour l'analyse de l'occupation de l'espace en zones urbaine et périurbaine présaharienne ; cas de Lag-houat (Algérie). Revue Télédétection ; Vol. 6, n²; pp. 177-190.

Benkouider et al. (2012) : Extraction de routes par classification supervisée et par réseaux de neurones artificiels à partir d'image spot: cas d'une ville Oasienne (Algérie); Revue Télédétection ; vol. 11 ; $\mathrm{n}^{\circ} 1$; pp. 237-249.

Conseil Général 'Loire' : Mise en place d'un SIG pour la gestion des équipements sur les routes départementales de la Loire ; http://www.cotita.fr/IMG/pdf_Mise_en_place_SIG_Loire_1.pdf 
Dupuis, Blaise (2005), Une approche géographique de la gestion de l'affichage en ville de Neuchâtel: logiques de localisation, stratégies des acteurs et partenariat public-privé ; Mémoire de diplôme universitaire ; Université de Neuchâtel, 2005.

Emery, Justin, Marilleau, Nicolas, Thevenin, Thomas, Martiny, Nadège (2014), Du comptage ponctuel à l'affectation par simulation multi-agents : Application à la circulation routière de la ville de Dijon; sagéo ; 2014 ; https://horizon.documentation.ird.fr/exl-doc/plei ns_textes/divers15-06/010064551.pdf

Mentfakh, T. ; et Si-Afif, A.-M. (2014), Réalisation d'un Système d'Information Géographique pour la signalisation du réseau routier : cas pratique la région de Mostaganem; mémoire de master en informatique-SIG; Faculté des Sciences Exactes \& de l'Informatique Département de Mathématiques et d'Informatique Filière Informatique ; université de Mostaganem ; 2014 ; URI: http://e-biblio.univ-mosta.dz/handle/123456789/9397

Moretti, S.-D. (2018), «la gestion et le partage des données des sentiers récréatifs canadiens - une nouvelle norme des données intégrée à un SIG Web»; Mémoire présenté pour l'obtention du grade Maître en sciences géographiques (M.Sc.), cheminement géomatique ; Département de Géomatique Appliquée, Faculté des lettres et sciences humaines, Université de Sherbrooke ; avril 2018,

Pellecuer, Luc, Roy, Jean-Philippe, St-Jacques, Michèle, Assaf, Gabriel (2009), Gestion du marquage routier: analyse des acquis et perspectives pour le Québec; Congrès AQTR 2009, https://www.researchgate.net/publication/271526432_GESTION_DU_MARQUAGE _ROUTIER_ANALYSE_DES_ACQUIS_ET_PERSPECTIVES_POUR_LE_QUEBEC 